\title{
THE ADVERSE EFFECT OF FORMALIN: A WARNING AGAINST MISHANDLING
}

C.B. Lian, W.C. Ngeow. The adverse effect of formalin: A warning against mishandling. Annal Dent Univ Malaya 2000; 7: 56-58.

\begin{abstract}
Formalin is a clear solution of $37 \%$ formaldehyde in water. It is used in dentistry as a disinfectant, antiseptic and mainly as tissue fixative for preserving biologic specimens for histopathologic examination. The human knowledge on systemic formaldehyde intoxication is inadequate as only few cases of formalin ingestion have been reported. This paper presents a brief communication of the adverse effect of formalin to the human tissue.
\end{abstract}

Key words: adverse effect, formaldehyde, formalin

\section{INTRODUCTION}

Formaldehyde is a toxic colourless, water soluble foul smelling gas that extremely irritates the upper airways. Its soluble form is called formalin and is commonly used in medicine and dentistry to preserve biopsy specimens and as an antiseptic agents in clinics (1).

Systemic formaldehyde intoxication has not been adequately characterised because of lack of number of reports on the effect of formalin ingestion. Several deaths had been reported (1-5). Those who survived experienced distal stomach scarring $(6,7)$.

A case has also been reported whereby formalin was used accidentally as irrigating solution during a wisdom tooth surgery instead of normal saline (8). This happened because the surgical face masks worn by the surgeons hindered the sense of smell. Moreover, the surfaces of the surgery were usually wiped with antiseptic containing formaldehyde, thus making it very difficult to locate the origin of formalin smell in the operating theatre. In addition, formalin at room temperature is crystal clear, the same as that of normal saline. Thus it can easily be mistaken as normal saline. Besides, the label on the formalin bottle had faded after repeated use and long term storage. The patient survived the formalin mishap without any systemic problem (8).

Formalin can enter the body by either ingestion or inhalation. There is little information available on the extent of formaldehyde absorption in mammals and there is little data concerning its conversion to formic acid and carbon dioxide in humans. The very few reports available demonstrated that formic acid accumulates markedly after the ingestion of formaldehyde. This conversion from formaldehyde to formic acid had been reported as rapid $(2,3,9)$.
Brief Communication

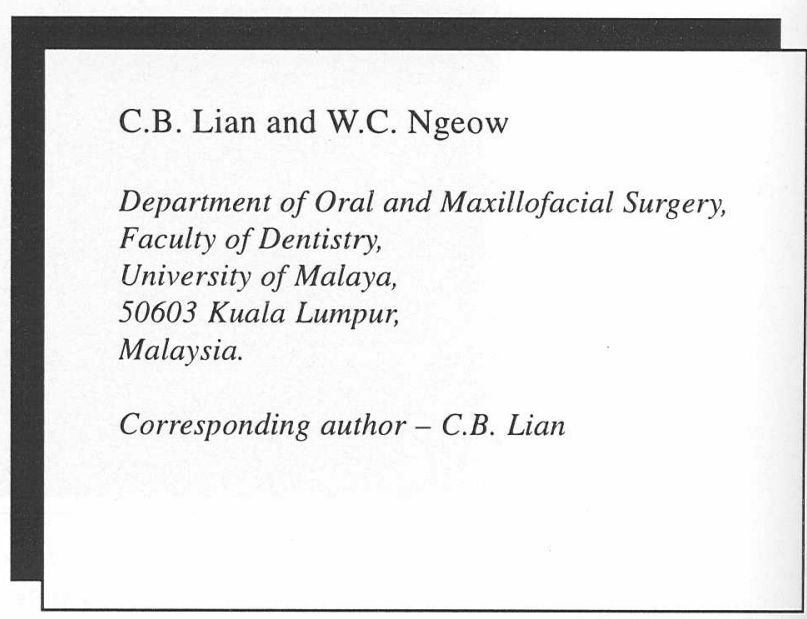

In the few reported cases of formalin poisoning, the patients presented with some of the following signs and symptoms in the emergency clinic.

\section{Clinical signs and symptoms}

The few signs and symptoms that has been reported following the ingestion of formalin are (3):

- severe abdominal pain

- retching

- development of seizures

- hypotension

- difficulty in breathing

These signs and symptoms result from biochemical, physiological and pathological changes in the body.

\section{Biochemical changes}

Formalin poisoning manifests the same end pathway as methanol poisoning. Methanol has been shown to be metabolised to formic acid with formaldehyde as a metabolic intermediate in humans (9). Although ingested formaldehyde may produce extensive pathologic changes in the gastrointestinal tract, sufficient formaldehyde can apparently be absorbed and metabolised rapidly to formic acid. Formic acid may accumulate rapidly in the blood to cause metabolic acidosis (2). Metabolic acidosis happens in part because of high plasma levels of formic acid acidosis, the main metabolite of formaldehyde and in part as a result of hyperlactatemia (1).

\section{Physiological changes}

Combination of hypovolaemia, vasodilation and a myocardial depressant effect of formalin has been reported to happen as a result of deliberate ingestion of 50-100 cc of formalin (5). The patient ended up with metabolic acidosis, gastric ulceration and circulatory shock two hours after ingestion of the poison. Development of seizures and disseminated intravascular coagulation have also been reported. 
Hypovolaemic shock, mainly secondary to the appearance of digestive tract haemorrhage, has been most often reported (10). The myocardial toxicity of formalin is another complication noted (1). Formalin also has direct oxidant action on red blood cells to cause acute intravascular hemolysis (11).

\section{Pathological changes}

The following organs may undergo degenerative changes following formalin ingestion:

- central nervous system

- kidney

- gastrointestinal tract

- liver

- heart

- lung

The clinical course may be marked by an initial profound central nervous system depression (3). The kidney may experience impairment in function, which may rapidly progress to acute renal failure and anuria (1).

The gastrointestinal tract, especially the stomach may undergo corrosive damage. Burns of the jejunum, ileum, and part of the colon including the sigmoid have been noted in autopsies (1). There may be perforating mucosal necrosis of the oesophagus (1). Hepatosplenomegaly with jaundice and congestion of the liver parenchyma have also been reported (1).

The heart may undergo fibrosis. This will cause cardiac insufficiency (1). The lung may become fibrosed, resulting in respiratory insufficiency and eventual acute respiratory distress syndrome (1). The subsequent course of formalin poisoning consists of multiple organ failure and death. The fatal dose of ingested formalin is about 50-100 millilitres (5).

\section{MANAGEMENT}

Rapid intubation and ventilation has been advocated as the immediate management in cases of formalin ingestion. The patient's blood pressure and urine output should be monitored and gastric lavage performed (2). Because formic acid accumulates rapidly following ingestion of formaldehyde, serious consideration should be given to dialysis procedures. The types of dialysis that can be given are:

- haemodialysis (1)

- peritoneal dialysis (9)

- haemofiltration (1).

Dialysis is a procedure for the removal of formaldehyde and formic acid from the blood by virtue of the difference in their rates of diffusion through an external semipermeable membrane or in the case of peritoneal dialysis, through the peritoneum.
Dialysis is effective in removing formic acid from the blood of humans (9). It is suggested that the removal of formic acid may be effective in combating the metabolic acidosis that may occur in systemic formaldehyde poisoning $(1,2,11)$.

Other treatment attempted consisted of infusion of $\mathrm{N}$-acetylcysteine in combination with hemodialysis (5). Basically, formaldehyde forms an adduct with reduced glutathione (GSH) forming the S-hydroxymethylgluthathione which is a substrate for the detoxification enzyme called the formaldehyde dehydrogenase. During acute toxicity, depletion of GSH occurs and hence formaldehyde (FA) can bind to other macromolecules leading to cellular damage. The obvious treatment is to increase GSH levels but unfortunately GSH does not enter hepatocytes where the detoxification enyzmes reside and hence administration of $\mathrm{N}$ acetylcysteine (NAC) which penetrates hepatocytes is given and therefore act as a substitute for glutathione or increase its synthesis (therefore restòring intracellular glutathione concentrations). GSH is also useful especially as an antioxidant in scavenging the reactive oxygen species occurring during the metabolism of FA. Moreover, by giving NAC, FA can then bind directly to it or the GSH (produced as a result of NAC treatment) and therefore reducing the conversion of FA to formic acid (12). This of course will reduce metabolic acidosis.

\section{CONCLUSION}

Formalin is a hazardous solution that needs special storage and handling. The support staffs should be aware of the adverse effect of this solution to the living tissue. Its usage should be strictly for disinfection, as antiseptic and as tissue fixative. It should never be stored close to other clear solution to avoid mishandling. If there is any doubt about the content of a bottle, the solution should be discarded. Poisoning by ingestion of formalin is lethal and must be treated at an early stage. The prognosis is linked to the extent of the caustic injuries and of the systemic effects.

\section{ACKNOWLEDGEMENT}

The authors would like to thank Dr. Salmaan H. InayatHussain of the Department of Biomedical Sciences, Faculty of Allied Health Sciences, Universiti Kebangsaan Malaysia for his invaluable advice in preparing this manuscript.

\section{REFERENCES}

1. Köppel C, Baudisch $\mathrm{H}$, Schneider V, Ibe $\mathrm{K}$ : Suicidal ingestion of formalin with fatal complications. Intensive Care Med, 1990; 16: 212 4. 
2. Eells JT, McMartin KE, Black K, Virayotha V, Tisdell RH, Tephly TR: Formaldehyde poisoning. Rapid metabolism to formic acid. JAMA, 1981; 246: $1237-8$.

3. Burkhart KK, Kulig WW, McMartin KE: Formate levels following a formalin ingestion. Vet Hum Toxicol, 1990; 32: 135-7.

4. Eichler HG, Hruby K, Katschnig MJ, Leinzinger P: Accidental fatal formaldehyde poisoning. Wien Klin Wochenschr, 1983; 95: 243-5.

5. Hilbert G, Gruson D, Bedry R, Cardinaud JP: Circulatory shock in the course of fatal poisoning by ingestion of formalin. Intensive Care Med, 1997; 23: 708 .

6. Kochhar R, Nanda V, Nagi B, Mehta SK: Formaldehyde-induced corrosive gastric cicatrization: case report. Hum Toxicol, 1986; 5: $381-2$.

7. Roy M, Calonje MA, Mouten R: Corrosive gastritis after formaldehyde ingestion. N Engl J Med, 1962; 266: 1248-9.
8. Lian CB, Ngeow WC: Formalin mishandling during wisdom tooth surgery. Oral Surg Oral Med Oral Pathol Oral Radiol Endod, 1998; 86: 630-1.

9. Mc Martin KE, Ambre JJ, Tephly TR: Methanol poisoning in human subjects: Role of formic acid accumulation in the metabolic acidosis. Am J Med, 1980; 68: 414-8.

10. Rajan N, Rahim R, Krishna Kumar S: Formic acid poisoning with suicidal intent: a report of 53 cases. Postgrad Med J, 1985; 61: 35-6.

11. Pun KK, Yeung CK, Chan TK: Acute intravascular hemolysis due to accidental formalin intoxication during hemodialsysis. Clinical Nephrology, 1984; 21: $188-90$.

12. Casanova M, Heck Hd'A. Further studies of the metabolic incorporation and covalent binding of inhaled [3H]- and [14C]formaldehyde in Fischer344 rats: effects of glutathione depletion. Toxicol Appl Pharmacol 1987; 89: 105-21. 